Journal of Applied Finance \& Banking, Vol. 11, No. 5, 2021, 95-119

ISSN: 1792-6580 (print version), 1792-6599 (online)

https://doi.org/10.47260/jafb/1155

Scientific Press International Limited

\title{
Market Trends and Options Trading: Viewpoint, Probability and Implications
}

\author{
Monica Guling Wu ${ }^{1}$, Hsinan $\mathrm{Hsu}^{2}$ and Janchung Wang ${ }^{3}$
}

\begin{abstract}
In Dow theory, market trends are classified as secular trends for long-term frames, primary trends for medium-term frames, and secondary trends for short-term frames. For the long and medium terms, they can consist of major bull (bear) markets and minor bear (bull) markets; for the short terms, they may have corrections and bear rallies. These definitions of market trends are not very helpful to options traders because in practice, options trading is often done on a short-term time frame and options have a unique property of time value. Even in a bull market, there is a possibility of losing all money for buying call options; in a bear market, there is a probability of earning money for buying call options. This inconsistency often troubles options traders deeply. From the viewpoint of options trading, we introduce a new concept of analyzing the market trends and propose new methods for estimating the probabilities of the market trends since at any time the future prices are unknown. By simplifying the market trends into three concepts of uptrend, downtrend, and neutral trend, it will have consistent implications for options trading.
\end{abstract}

JEL classification numbers: C13, G10, G13.

Keywords: Market trends, Options trading, Trend probability, Estimation methods, Trading implications.

${ }^{1}$ College of Finance and Banking, National Kaohsiung University of Science and Technology, Taiwan, R.O.C.

2 Department of Finance, Feng Chia University, Taiwan, R.O.C.

3 Department of Money and Banking, National Kaohsiung University of Science and Technology, Taiwan, R.O.C.

Article Info: Received: July 30, 2021. Revised: August 24, 2021.

Published online: August 30, 2021. 


\section{Introduction}

A market trend is a perceived tendency of the price level of a financial (e.g., stock) market to move in a particular direction over time. According to the technical analysis of the conventional Dow theory, market trends are classified as:

a) secular trends for long-term frames,

b) primary trends for medium-term frames, and

c) secondary trends for short-term frames (Edwards, Magee, and Bassetti, 2018).

Furthermore, a period of an upward market trend is called a bull market and a downward market trend is called a bear market (Stevens, 2002). Therefore, for the long and medium terms, they consist of a (or several) major bull market(s) and a (or several) minor bear market(s), or a (or several) major bear market(s) and a (or several) minor bull market(s); for the short terms, they may have corrections and bear rallies. The time frames of trend periods are different: Secular trends may last 5 to 25 years, primary trends one year or more, and secondary trends a few weeks or few months (Wikipedia, 2019).

However, these definitions of the market trends are not very helpful to the options traders because in practice, options trading is often short-term frame ${ }^{4}$ and options have a unique property of time value. Thus, even in a long or medium term of bull markets, there is a possibility of losing all money for buying calls and a possibility of earning big money for buying puts. On the contrary, in bear markets, there is a probability of losing all money for buying puts and a possibility of earning big money for buying calls. These problems often puzzle many options traders. In addition to the incongruencies within the definitions of the conventional trends, other reasons may be responsible for this puzzle: Firstly, adopting technical indicators to determine the market trends often shows a lag phenomenon due to using historical data. Secondly, the market trends often show in hindsight since security prices in the future are not easy to predict. ${ }^{5}$ Thus, a certain type of trend can only be presented in probability. Thirdly, the decay of an option's time value is irrelevant to the market trends.

In this paper, the usefulness of current techniques for analyzing the classical definitions of market trends are first examined by using the strategy of trend identification. We find that when the bull markets are identified, only $51.24 \%$ of buying call options with a wide range of moneyness obtain positive returns, while $48.76 \%$ have negative returns. Similarly, concerning the bear markets, only $49.45 \%$ of buying put options with a wide range of moneyness obtain positive returns, and

4 This is evidenced by large trading volumes for weekly and near monthly options, and small trading volumes for far month options. For example, according to TAIFEX statistics, on August 24, 2020, the TXO (the most active trading option in Taiwan) trading volumes for all maturities are as follows: 202008w4 (weekly options): 495,469 contracts, 202009 (the nearest monthly options): 81,415 contracts, 202010 (the second monthly options): 2,530 contracts, 202011 (the third monthly options): 365 contracts, 202012 (the first quarterly options): 451 contracts, and 202103 (the second quarterly options): 76 contracts.

5 See "Market Trend", https://capital.com/market-trend-definition, or Wikipedia (2019), "Market Trend", https://en.wikipedia.org/wiki/Market_trend. 
$50.55 \%$ have negative returns. Thus, the classical view of the market trends is questionable for options trading and must be redefined to solve the doubts.

To have consistent implications for options trading, this paper is based on the viewpoint of options trading to simplify the market trends into three concepts: uptrend, downtrend, and neutral (sideways) trend. A trend can only be determined in hindsight as the future prices are unknown, hence we propose two estimation methods for determining the probabilities of the market trends under this new point of view and discuss the important implications to options trading in this study.

The remaining part of this article is organized as follows: Section 2 is a review of the classical view of the market trends and provides an empirical test to examine whether the technical analyses are helpful in determining options trading. Section 3 illustrates the central theme of this paper, namely, a new view of the market trends for options trading, including the concept as well as the theoretical and empirical methods for estimating the probabilities of the market trends. Section 4 discusses the implications of this new view of the market trends and how it affects options trading. At last, Section 5 presents the conclusions of this paper.

\section{The Classical View of Market Trends and Options Trading}

\subsection{A classical view of the market trends}

A market trend is the perceived direction of price movements over a particular period which can be applied to all assets and all markets, such as stocks, bonds, foreign exchange, and futures, where there is a fluctuation of prices. The movement is analyzed by comparing the historical price movement against the current price. Simply put, a market trend is used to describe the upward or downward movement of prices over time. According to the Dow theory, when prices experience a series of successively higher peaks and higher troughs, it is an upward trend; on the contrary, if there are consistently lower peaks and lower troughs, it is a downward trend. In financial markets, an upward trend is called a bull market which is a period of generally rising prices and is commonly defined as prices rise by $20 \%$; on the other hand, a downward trend is called a bear market which is a general decline of $20 \%$ or more on prices from recent highs over a sustained period (Yardeni, Abbott, and Quintana, 2020). When the price has been experiencing neither an upward trend nor a downward trend, it is called a sideways trend (Stevens, 2002), in which the peaks and troughs continue to be constant. It is generally a result of the price traveling between strong levels of support and resistance, and when it ends, an uptrend or a downtrend may begin.

In terms of the time frame, trends can be long-, medium-, or short-term. For the long and medium terms, they can consist, respectively, of a (or several) major bull market(s) and a (or several) minor bear market(s), or a (or several) major bear market(s) and a (or several) minor bull market(s). For the short terms, the trend may have market corrections and/or bear market rallies. According to the technical analysis of the conventional Dow theory, market trends are classified as: a secular trend for long-term frames, a primary trend for medium-term frames, and a 
secondary trend for short-term frames (Edwards, Magee, and Bassetti, 2018). The time frames of these three trend periods are vastly different: Secular trends may last 5 to 25 years, primary trends twelve months or more, and secondary trends a couple of weeks or a few months (Wikipedia, 2019). A secular market trend consists of a series of primary trends, for example, a secular bull market consists of larger bull markets and smaller bear markets; a secular bear market consists of large bear markets and smaller bull markets. In a secular bull market, the prevailing trend is bullish or upward moving, while in a secular bear market, the prevailing trend is bearish or downward moving. A secondary trend is a short-term change in the price direction within a primary trend. When prices decline by $5 \%$ to $20 \%$ or so within a short duration, it is called a market correction which represents the reversal of a bull market. In contrast, when prices temporarily increase (e.g., 10\% or 20\%) after a sustained decline, it is called a bear market rally or a dead cat bounce which represents the temporary reversal of a bear market.

\subsection{Is classical view of market trends useful to options trading?}

The definitions of classical market trends as mentioned above may not be very helpful to options traders in reality because most option transactions are often shortterm oriented, as evidenced by large volumes for weekly and monthly options. Even in a long or medium term of bull markets, there is a probability of losing all money for buying call options and a probability of earning big money for buying put options. On the contrary, in bear markets, there is a probability of losing all money for buying put options and a probability of earning big money for buying call options. Options traders are often puzzled with this experience by using classical view of stock market trends. To clarify this area of knowledge, the technical indicators used to identify the prevailing market trend are tested first as to whether they can improve the performance of a simple buying strategy on options. If the answer is negative, then there is the justification to adopt a new view of market trends for options trading.

\subsection{Methodology}

A technical analysis is often used in the stock market to identify the prevailing market trend (Stevens, 2002), thereby determining the timing of buying or selling securities to make profits. Here, an empirical test is employed to examine whether the identified market trend by technical analysis is indeed helpful to options trading. If the technical indicator shows an entry signal, it is beneficial to buy call options; if the indicator shows an exit signal, then it is the timing to close out the call positions and simultaneously buy put options. The performance of this strategy will be compared with that of the naive option trading strategy, i.e., purely buying calls or puts.

In this paper, the TAIEX options (TXO) are used to compare the realized returns of both two option trading strategies because the TXO are highly liquid with large trading volumes and are quite efficient. Two types of expiration options (i.e., 
monthly options and weekly options) ${ }^{6}$ are employed to compare whether the holding periods can influence the performance of options trading. In Taiwan, each weekly option begins trading on every Wednesday at 8:45 AM and expires at 13:30 PM on the next Wednesday, except holidays. ${ }^{7}$ In practice, the opening prices are difficult to know, thus for weekly options, it is reasonable to assume that the holding period is from one Wednesday's close to the next Wednesday's settlement, or seven days in total. As for monthly options, the duration is not the same: Some last for two months and others may last as long as for nine months. For the convenience of sampling and comparison, the holding period of monthly options is four weeks.

The underlying asset of the TXO is the Taiwan Capitalization Weighted Stock Index (TAIEX). One of the most common technical indicators, a Simple Moving Average $(\mathrm{SMA})^{8}$, is here used to identify the market trends. The sampling period of the TXO covers December 2001 through December 2019 for monthly options and July 2013 through December 2019 for weekly options. ${ }^{9}$ The price data of the weekly options and monthly options and the daily data of the TAIEX are retrieved from the Taiwan Futures Exchange (TAIFEX) and Taiwan Economic Journal (TEJ), respectively.

\subsection{Results}

\subsubsection{The market trend of the TAIEX}

First, the market trend of the TAIEX during the whole sampling period is shown in Figure 1. It reveals that the secular market trend of the Taiwan stock market has shown an upward trend, a secular bull market, over the past two decades which consists of larger bull markets and smaller bear markets. Table 1 shows the descriptive statistics for the TAIEX, the short-term moving average MA5, and the long-term moving average MA120. There are 4460 observations in our sampling period, which displays an average of 7840 , with a minimum of 3850 , a maximum of 12122 , and a standard deviation of 26.84 of the TAIEX. The mean of the shortterm MA5 is close to that of the TAIEX, while the mean of the long-term MA120 is about 100 points lower than that of the TAIEX.

6 Since the trading volumes of longer maturity options (e.g., quarter options) are relatively small and may have no transactions for some selected strike prices, they were excluded from the sampling.

7 If Wednesday comes upon a non-trading day, the listing of options will delay until the trading day.

8 The SMA5 and SMA120 are used to present a short-term and a long-term moving average, respectively. When there is a crossover point, it is a signal to buy in or sell out the options. Specifically, when SMA5 is going from below SMA120, through it, and then above it, it is called a golden cross. Hence, it is advantageous to buy call options. On the contrary, when SMA5 is going from above SMA120, through it, and then below it, it is called a death cross. In this case, it is advantageous to liquidate the current positions of calls and simultaneously buy put options. A golden cross indicates a long-term bull market going forward, while a death cross signals a long-term bear market. Both refer to the solid confirmation of a long-term trend by the occurrence of a short-term moving average crossing over a major long-term moving average.

9 In Taiwan, the monthly options and weekly options are launched in December 2001 and July 2013, respectively. 
The Market Trend of TAIEX

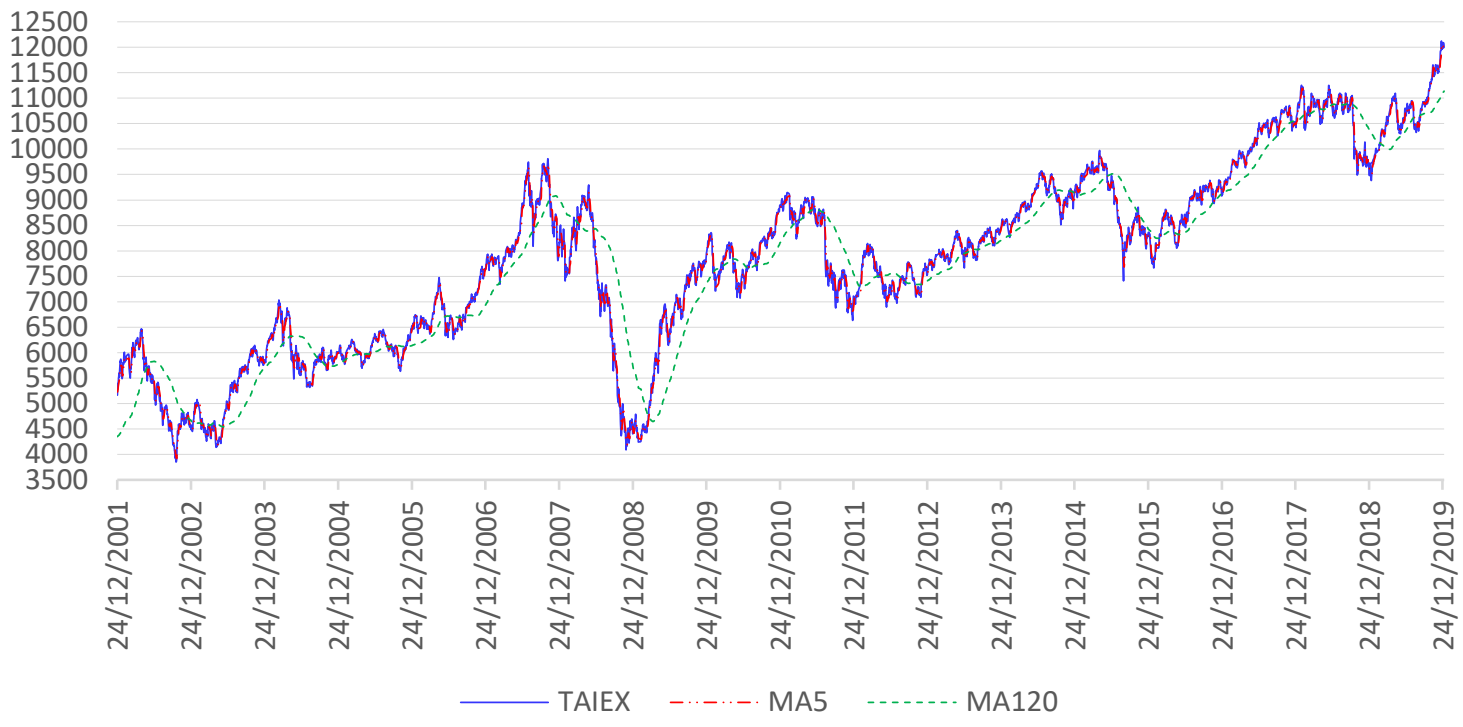

Figure 1: The market trend of the TAIEX

Note. In this figure, the TAIEX is the Taiwan Capitalization Weighted Stock Index which covers from Dec. 24, 2001 to Dec. 31, 2019. The MA5 and MA120 represent the 5-day and the 120-day simple moving averages of the TAIEX which were calculated from Dec. 18, 2001 and July 2, 2001 respectively, to Dec. 31,2019 . There exist some crossover points which are called golden crosses, when MA5 is going through and above MA120, and death crosses, when MA5 is going through and below MA120. Since the sampling period is quite long, the short-term MA5 and the TAIEX curves appear to overlap.

Table 1: Descriptive statistics of the TAIEX

\begin{tabular}{|c|c|c|c|}
\hline & TAIEX & MA5 & MA120 \\
\hline Mean & 7840.06 & 7837.00 & 7746.62 \\
\hline Std. dev. & 26.84 & 26.80 & 26.06 \\
\hline Median & 7903.23 & 7911.36 & 7827.95 \\
\hline Min. & 3850.04 & 3919.39 & 4349.66 \\
\hline Max. & 12122.45 & 12043.934 & 11139.536 \\
\hline Skewness & -0.03 & -0.03 & -0.04 \\
\hline Kurtosis & -0.71 & -0.72 & -0.83 \\
\hline Observations & 4460 & 4460 & 4460 \\
\hline
\end{tabular}




\subsubsection{The observed return distributions of the TXO}

Next, the observed returns distributions of the weekly TXO and monthly TXO are presented, respectively, using the naïve strategy (i.e., buying options at the beginning of contracts and closing the positions at their expirations) in the whole sampling period. In the Taiwan options market, the range between two consecutive strike prices is 50 (100) points for the weekly (monthly) options. For sufficient coverage of the moneyness of options and for considering the options trading volume, the range of strike price included in the test is 200 (400) points above and below the ATM (at-the-money) for the weekly (monthly) options. Five returns of options with different strike price are calculated in each sampling date by using the method of arithmetic return instead of that of logarithmic return because options may expire worthless. ${ }^{10}$

Table 2 and Table 3 report the return distributions of the weekly TXO and monthly TXO, respectively. In the sampling period, the number of weekly options and monthly options are 332 and 217, respectively. From both tables, for call options, weekly or monthly, the percentages of losing all money $(R=-1)$ all increase with the strike price; the percentages of having positive profits all decrease with the strike price. That is, the deeper the calls are out-of-the-money (OTM), the more likely they expire worthless; the deeper the calls are in-the-money (ITM), the more likely they expire with positive profits. For instance, in Panel A of Table 2, the percentage of losing all money is only $9.64 \%$ for the weekly ITM2 calls but as high as $90.66 \%$ for the weekly OTM2 calls; the percentage of having positive profit is $56.93 \%$ for the weekly ITM 2 calls but only $8.43 \%$ for the weekly OTM 2 calls. The same is true for the weekly put options in Panel B of Table 2. In short, the deeper the options are ITM, the more likely they will have positive profits.

${ }^{10}$ In financial literature, security return is often calculated by using the method of continuously compounded return (i.e., log return). However, if the log return for options' holding up to expiration is used, it will result in a serious problem because options may expire worthless. In this case, $\ln (0) \rightarrow-\infty$. Therefore, neither the mean nor the standard deviation of the option return is obtainable. 
Table 2: The return distribution (probabilities) of the weekly TXO

\begin{tabular}{|c|c|c|c|c|c|c|c|}
\hline \multirow{2}{*}{ Strike price } & \multirow{2}{*}{$\begin{array}{c}\text { Total } \\
\text { no. }\end{array}$} & $\begin{array}{c}\text { Positive profit } \\
(\boldsymbol{R}>\mathbf{0})\end{array}$ & \multicolumn{2}{c|}{$\begin{array}{c}\text { No profit } \\
(-1<\boldsymbol{R})\end{array}$} & \multicolumn{2}{|c|}{$\begin{array}{c}\text { Lose all } \\
(\boldsymbol{R}=-\mathbf{1})\end{array}$} \\
\cline { 2 - 8 } & No. & \% & No. & \% & No. & \% \\
\hline \multicolumn{8}{|c|}{ Panel A: Call options } \\
\hline ITM2 & 332 & 189 & $56.93 \%$ & 111 & $33.43 \%$ & 32 & $9.64 \%$ \\
\hline ITM1 & 332 & 173 & $52.11 \%$ & 91 & $27.41 \%$ & 68 & $20.48 \%$ \\
\hline ATM & 332 & 134 & $40.36 \%$ & 57 & $17.17 \%$ & 141 & $42.47 \%$ \\
\hline OTM1 & 332 & 73 & $21.99 \%$ & 15 & $4.52 \%$ & 244 & $73.49 \%$ \\
\hline OTM2 & 332 & 28 & $8.43 \%$ & 3 & $0.90 \%$ & 301 & $90.66 \%$ \\
\hline & \multicolumn{7}{|c|}{ Panel B: Put options } \\
\hline ITM2 & 331 & 123 & $37.16 \%$ & 174 & $52.57 \%$ & 34 & $10.27 \%$ \\
\hline ITM1 & 332 & 108 & $32.53 \%$ & 133 & $40.06 \%$ & 91 & $27.41 \%$ \\
\hline ATM & 332 & 82 & $24.70 \%$ & 57 & $17.17 \%$ & 193 & $58.13 \%$ \\
\hline OTM1 & 332 & 52 & $15.66 \%$ & 15 & $4.52 \%$ & 265 & $79.82 \%$ \\
\hline OTM2 & 332 & 27 & $8.13 \%$ & 5 & $1.51 \%$ & 300 & $90.36 \%$ \\
\hline
\end{tabular}

Note. The ATM (at-the-money) is defined as the strike price closest to the current TAIEX. The range between two consecutive strike prices is 100 points for weekly options. For example, if the ATM is 8000, the ITM (in-the-money) and OTM (out-of-the-money) calls are as follows: ITM2 7800, ITM1 7900, OTM1 8100 and OTM2 8200. The formula of the return, taking the call option as an example, is $R_{c}=\left(C_{T}-C_{0}\right) / C_{0}$, where $C_{0}$ and $C_{T}$ is the call price at time $\mathrm{t}=0$ and $T$, respectively. Each row presents the number and the percentage of options trading having a positive profit, a negative profit or losing all money. The three percentage columns $(\%)$ are the concept of probabilities.

These conclusions are also true for the monthly options. For example, in Table 3, the percentage to win is $49.77 \%$ for the monthly ITM1 calls and up to $53.46 \%$ for the monthly ITM2 calls; the percentage of having positive profits for the monthly ITM puts is more than $30 \%$ but less than that for the monthly ITM calls. When considering the non-profit side, the chance of losing all money for the deeper monthly ITM puts is $19.63 \%$ which is almost two times that of the weekly puts, $10.27 \%$. In short, the deeper the options are OTM, the more likely they expire nonprofitable. The results in both Table 2 and Table 3 indicate that 70 90\% (60 85\%) of the weekly options (monthly options) expire worthless, which are also consistent with Summa's (2003) empirical results and Hsu's (2015) theoretical results. 
Table 3: The return distribution (probabilities) of the monthly TXO

\begin{tabular}{|c|c|c|c|c|c|c|c|}
\hline \multirow{2}{*}{ Strike price } & \multirow{2}{*}{$\begin{array}{c}\text { Total } \\
\text { no. }\end{array}$} & $\begin{array}{c}\text { Positive profit } \\
(\boldsymbol{R}>\mathbf{0})\end{array}$ & \multicolumn{2}{c|}{$\begin{array}{c}\text { No profit } \\
(-\mathbf{1}<\boldsymbol{R})\end{array}$} & \multicolumn{2}{c|}{$\begin{array}{c}\text { Lose all } \\
(\boldsymbol{R}=-\mathbf{0})\end{array}$} \\
\cline { 3 - 8 } & No. & \% & No. & $\%$ & No. & \% \\
\hline \multicolumn{8}{|c|}{ \%: Call options } \\
\hline ITM2 & 217 & 116 & $53.46 \%$ & 71 & $32.72 \%$ & 30 & $13.82 \%$ \\
\hline ITM1 & 217 & 108 & $49.77 \%$ & 57 & $26.27 \%$ & 52 & $23.96 \%$ \\
\hline ATM & 217 & 97 & $44.70 \%$ & 23 & $10.60 \%$ & 97 & $44.70 \%$ \\
\hline OTM1 & 216 & 62 & $28.70 \%$ & 21 & $9.72 \%$ & 133 & $61.57 \%$ \\
\hline OTM2 & 215 & 34 & $15.81 \%$ & 8 & $3.72 \%$ & 173 & $80.47 \%$ \\
\hline & \multicolumn{7}{|c|}{ Panel B: Put options } \\
\hline ITM2 & 214 & 82 & $38.32 \%$ & 90 & $42.06 \%$ & 42 & $19.63 \%$ \\
\hline ITM1 & 216 & 75 & $34.72 \%$ & 59 & $27.31 \%$ & 82 & $37.96 \%$ \\
\hline ATM & 217 & 60 & $27.65 \%$ & 38 & $17.51 \%$ & 119 & $54.84 \%$ \\
\hline OTM1 & 217 & 41 & $18.89 \%$ & 13 & $5.99 \%$ & 163 & $75.12 \%$ \\
\hline OTM2 & 217 & 18 & $8.29 \%$ & 13 & $5.99 \%$ & 186 & $85.71 \%$ \\
\hline
\end{tabular}

Note. The ATM (at-the-money) is defined as the strike price closest to the current TAIEX. The range between two consecutive strike prices is 200 points for monthly options. For example, if the ATM is 8000, the ITM (in-the-money) and OTM (out-of-the-money) calls are as follows: ITM2 7600, ITM1 7800 , OTM1 8200 and OTM2 8400. The formula of the return, taking the call option as an example, is $R_{c}=\left(C_{T}-C_{0}\right) / C_{0}$, where $C_{0}$ and $C_{T}$ is the call price at time $\mathrm{t}=0$ and $T$, respectively. Each row presents the number and the percentage of options trading having a positive profit, a negative profit, or losing all money. The three percentage columns (\%) are the concept of probabilities.

\subsubsection{Market trend and performance of options trading}

It is interesting to know whether the identified market trends using a technical indicator can improve the performance of options trading. To answer this important question, one of the most common technical indicators, a Simple Moving Average (SMA), is used to identify the daily trends of the TAIEX (see footnote 8 for the illustration). After every entrance and exit date have been identified (i.e., the beginning and the end of every bull and bear market are identified), the following strategy called "with trend identification strategy" is used: In a bull (bear) trend, call (put) options are bought on every sampling date until their expiration dates or the end of this trend, whichever comes first. Then, the average returns of trading options between the strategy of without trend identification (i.e., naïve strategy) and the strategy of with trend identification are compared.

Table 4 and Table 5 present the results of the average returns of the two trading strategies for the weekly options and the monthly options, respectively. Both tables show that the average returns on the weekly and monthly calls of all moneyness 
using the naïve strategy are all positive and generally increase with strike price ${ }^{11}$, which are consistent with the prediction of Coval and Shumway (2001) $)^{12}$ and Hsu (2015); on the contrary, the average returns on the weekly and monthly puts of all moneyness using the naïve strategy are all negative. In contrast, under the strategy of with trend identification, the results reveal that both the average returns on the weekly and monthly options of all moneyness are all positive, and the OTM options seem to outperform the ITM options.

Table 4: The returns of the weekly TXO

\begin{tabular}{|c|c|c|c|c|}
\hline \multirow{2}{*}{ Strike price } & \multicolumn{3}{|c|}{$\begin{array}{c}\text { Without trend identification } \\
\text { (Naïve) strategy } \\
\left(\overline{\boldsymbol{\chi}}_{\mathbf{1}}\right)\end{array}$} & $\begin{array}{c}\text { With trend } \\
\text { identification strategy } \\
\left(\overline{\boldsymbol{\chi}}_{\mathbf{2}}\right)\end{array}$ \\
\cline { 2 - 5 } & $\overline{\boldsymbol{R}}_{\boldsymbol{c}}$ & $\overline{\boldsymbol{R}}_{\boldsymbol{p}}$ & $\overline{\boldsymbol{R}}_{\boldsymbol{a v g}}$ & $\overline{\boldsymbol{R}}_{\text {trend }}$ \\
\hline ITM2 & 0.0870 & -0.0823 & 0.0024 & 0.0427 \\
\hline ITM1 & 0.1371 & -0.1106 & 0.0133 & 0.0692 \\
\hline ATM & 0.1791 & -0.1619 & 0.0086 & 0.1039 \\
\hline OTM1 & 0.4754 & -0.1509 & 0.1623 & 0.3721 \\
\hline OTM2 & 0.6466 & -0.1112 & 0.2677 & 0.3483 \\
\hline Mean & 0.3051 & -0.1234 & 0.0908 & 0.1872 \\
\hline Sta. dev. & 0.2436 & 0.0326 & 0.1194 & 0.159 \\
\hline Variance & 0.0593 & 0.0011 & 0.0142 & 0.0255 \\
\hline No. of obs. $(n)$ & 332 & 332 & 332 & 332 \\
\hline$t$-value & -0.9047 & $4.2641 * *$ & 1.0815 & \\
\hline
\end{tabular}

Note. $\bar{R}_{c}$ and $\bar{R}_{p}$ are the average returns on call options and put options, respectively, under the naïve strategy. The option returns are calculated by using arithmetic returns, e.g., $R_{c}=\left(C_{T}-\right.$ $\left.C_{0}\right) / C_{0}$, where $C_{0}$ and $C_{T}$ are the call prices at time $t=0$ and $T$, respectively. $\bar{R}_{\text {avg }}$ is the average of $\bar{R}_{c}$ and $\bar{R}_{p}$. $\bar{R}_{\text {trend }}$ represents the average option returns for the strategy of with trend identification (i.e., in a bull trend, the options are calls; in a bear trend, the options are puts). The test determines whether the average returns for the naïve strategy are significantly different from that for the trend identification strategy. Mathematically, the null hypothesis is $\mathrm{H}_{0}: \bar{\chi}_{2}-\bar{\chi}_{1}=0$, vs. $\mathrm{H}_{1}: \bar{\chi}_{2}-\bar{\chi}_{1} \neq 0$, where $\bar{\chi}_{1}$ is the average return $\left(\bar{R}_{c}, \bar{R}_{p}\right.$, or $\left.\bar{R}_{\text {avg }}\right)$ for strategy 1 (without trend identification), and $\bar{\chi}_{2}$ is the average return for strategy 2 (with trend identification). The $* * *, * *, *$ indicate the statistical significance at the $1 \%, 5 \%$, and $10 \%$ levels, respectively. The numbers shown in parentheses are the $p$-values.

${ }^{11}$ For large strikes of monthly calls (OTM), the returns decrease with the strike prices.

12 The Coval and Shumway (2001) propositions only deal with the range of security prices for which the option is in-the-money. 
In Table 4 , the means of the returns for the naïve strategy on the weekly calls $\left(\bar{R}_{c}\right)$ and puts $\left(\bar{R}_{p}\right)$ are 0.3051 and -0.1234 , respectively, with an average $\left(\bar{R}_{\text {avg }}\right)$ of 0.0908; the mean of the returns for the with trend identification strategy on the weekly options $\left(\bar{R}_{\text {trend }}\right)$ is 0.1872 . The results of $t$-statistics show that both $\bar{R}_{c}$ and $\bar{R}_{\text {avg }}$ are insignificantly different from $\bar{R}_{\text {trend }}$, but $\bar{R}_{p}$ is significantly different from $\bar{R}_{\text {trend }}$ at $\alpha=5 \%$ level, indicating that the trend identification strategy does not outperform the naïve strategy. Although $\bar{R}_{p}$ is significantly different from $\bar{R}_{\text {trend }}$, it is the nature of put option returns, which are negative, and it is also consistent with the literature as was mentioned in the above section. If traders were to sell put options, $\bar{R}_{\text {trend }}$ would not be significantly different from $\bar{R}_{p}$.

Table 5: The returns of the monthly TXO

\begin{tabular}{|c|c|c|c|c|}
\hline \multirow{2}{*}{ Strike price } & \multicolumn{3}{|c|}{$\begin{array}{c}\text { Without trend identification } \\
\text { (Naïve) strategy } \\
\left(\overline{\boldsymbol{\chi}}_{\mathbf{1}}\right)\end{array}$} & $\begin{array}{c}\text { With trend } \\
\text { identification strategy } \\
\left(\overline{\boldsymbol{\chi}}_{\mathbf{2}}\right)\end{array}$ \\
\cline { 2 - 5 } & $\overline{\boldsymbol{R}}_{\boldsymbol{c}}$ & $\overline{\boldsymbol{R}}_{\boldsymbol{p}}$ & $\overline{\boldsymbol{R}}_{\boldsymbol{a v g}}$ & $\overline{\boldsymbol{R}}_{\text {trend }}$ \\
\hline ITM2 & 0.1276 & -0.1578 & -0.0151 & 0.3994 \\
\hline ITM1 & 0.1796 & -0.2039 & -0.0122 & 0.5129 \\
\hline ATM & 0.3006 & -0.2252 & 0.0377 & 0.7205 \\
\hline OTM1 & 0.4850 & -0.3023 & 0.0913 & 0.9534 \\
\hline OTM2 & 0.4368 & -0.4287 & 0.0040 & 0.9041 \\
\hline Mean & 0.3059 & -0.2636 & 0.0212 & 0.6989 \\
\hline Sta. dev. & 0.1557 & 0.1061 & 0.0445 & 0.2407 \\
\hline Variance & 0.0242 & 0.0113 & 0.0020 & 0.0579 \\
\hline No. of obs. $(n)$ & 217 & 217 & 217 & 217 \\
\hline$t$-value & $3.0594 * *$ & $8.1763 * * *$ & $6.1849 * * *$ & \\
\hline & $(0.0156)$ & $(0.0000)$ & $(0.0035)$ & \\
\hline
\end{tabular}

Note. $\bar{R}_{c}$ and $\bar{R}_{p}$ are the average returns on call options and put potions, respectively, under the naïve strategy. The option returns are calculated by using arithmetic returns, e.g., $R_{c}=\left(C_{T}-\right.$ $\left.C_{0}\right) / C_{0}$, where $C_{0}$ and $C_{T}$ are the call prices at time $t=0$ and $T$, respectively. $\bar{R}_{\text {avg }}$ is the average of $\bar{R}_{c}$ and $\bar{R}_{p} . \bar{R}_{\text {trend }}$ represents the average option returns for the strategy of with trend identification (i.e., in bull trends, the options are calls; in bear trends, the options are puts). The test determines whether the average returns for the naïve strategy are significantly different from that for the trend identification strategy. Mathematically, the null hypothesis is $H_{0}: \bar{\chi}_{2}-\bar{\chi}_{1}=0$, vs. $\mathrm{H}_{1}: \bar{\chi}_{2}-\bar{\chi}_{1} \neq 0$, where $\bar{\chi}_{1}$ is the average return $\left(\bar{R}_{c}, \bar{R}_{p}\right.$, or $\bar{R}_{\text {avg }}$ ) for strategy 1 (without trend identification), and $\bar{\chi}_{2}$ is the average return for strategy 2 (with trend identification). The ***,**, * indicate the statistical significance at the $1 \%, 5 \%$, and $10 \%$ levels, respectively. The numbers shown in parentheses are the $p$-values. 
In Table 5 , the mean of the returns for the naïve strategy on the monthly calls $\left(\bar{R}_{c}\right)$ and puts $\left(\bar{R}_{p}\right)$ are 0.3059 and -0.2636 , respectively, with an average $\left(\bar{R}_{\text {avg }}\right)$ of 0.0212; the mean of the returns for the with trend identification strategy on the monthly options $\left(\bar{R}_{\text {trend }}\right)$ is 0.6989 . The results of $t$-tests show that $\bar{R}_{c}, \bar{R}_{p}$, and $\bar{R}_{\text {avg }}$ are all significantly different from $\bar{R}_{\text {trend }}$ at $\alpha=5 \%$ level, indicating that the trend identification strategy does outperform the naïve strategy for long-term options.

In sum, Table 4 and Table 5 show that trading options with the trend identification strategy does improve the performance for long-term option returns, but not for the short-term option returns. ${ }^{13}$

\subsubsection{Conditional probabilities of positive profits using conventional trend identification}

The reason for trading options with the trend identification strategy improves the performance for long-term options return may be due to the time $\operatorname{lag}^{14}$ and the unique property of time value for options ${ }^{15}$. These two factors of the time lag and the time value for long-term options are not as serious as for short-term options. Thus, it is of interest to know how often the options traders will win the game for trading long-term options when applying the conventional trend identification strategy.

Table 6 presents the conditional return distributions for the monthly TXO given that trends are identified. The strategy is that if a bull market is identified, then buying call options of all selected moneyness is adopted during this period; if a bear market is identified, then buying put options of all selected moneyness is applied during this period. Both Panels A and B of Table 6 reveal that the conditional numbers and the percentages of having a positive profit decrease from the ITM options to the OTM options given that bull markets or bear markets are identified. For example, the conditional numbers (percentages) of having a positive profit decrease from 99 $(70.21 \%)$ to $32(22.86 \%)$ in the bull markets and from $48(64.86 \%)$ to $17(22.37 \%)$ in the bear markets.

On the contrary, the conditional numbers and the percentages of having a negative profit increase from the ITM options to the OTM options given that bull markets or bear markets are identified. For example, the conditional numbers (percentages) of having a negative profit increase from $42(29.79 \%)$ to $108(77.14 \%)$ in the bull

13 More recently, some empirical studies test whether technical indicators can improve the performance of options trading. For example, Lai (2020) applies RSI and MACD and Tu (2020) uses KD and MA to test the usefulness of technical indicators in trading TXO within different time frames. The results of their studies are also consistent with our results. That is, options trading with a trend identification strategy will improve the performance for long-term option returns to some extent, but not for the short-term option returns.

14 Because a technical indicator uses the data of past prices to identify the trends, the identified trend must have a time lag behind the true trend.

15 Option's time value will decay as time elapses and the decay rate is more rapid for weekly options. This phenomenon is irrelevant to the bullish or bearish market. 
markets and from $26(35.14 \%)$ to $59(77.63 \%)$ in the bear markets. Options traders should be surprised with these results: That the percentages of options trading are so largely unprofitable, especially for the OTM options, given that market trends had been identified. Therefore, the usefulness of the classical view of market trends towards trading options is here criticized.

Table 6: The conditional return distributions (probabilities) of the monthly TXO given that trends are identified

\begin{tabular}{|c|c|c|c|c|c|}
\hline \multirow[t]{2}{*}{ Strike price } & \multirow{2}{*}{$\begin{array}{c}\text { Total } \\
\text { no. }\end{array}$} & \multicolumn{2}{|c|}{$\begin{array}{l}\text { Positive profit } \\
(\mathbf{R}>0)\end{array}$} & \multicolumn{2}{|c|}{$\begin{array}{c}\text { Negative profit } \\
(R \leq 0)\end{array}$} \\
\hline & & No. & $\%$ & No. & $\%$ \\
\hline \multicolumn{6}{|c|}{ Panel A: Bull market (buying calls) } \\
\hline ITM2 & 141 & 99 & $70.21 \%$ & 42 & $29.79 \%$ \\
\hline ITM1 & 141 & 93 & $65.96 \%$ & 48 & $34.04 \%$ \\
\hline ATM & 141 & 84 & $59.57 \%$ & 57 & $40.43 \%$ \\
\hline OTM1 & 141 & 53 & $37.59 \%$ & 88 & $62.41 \%$ \\
\hline OTM2 & 140 & 32 & $22.86 \%$ & 108 & $77.14 \%$ \\
\hline Average & & & $51.24 \%$ & & $48.76 \%$ \\
\hline \multicolumn{6}{|c|}{ Panel B: Bear market (buying puts) } \\
\hline TM2 & 74 & 48 & $64.86 \%$ & 26 & $35.14 \%$ \\
\hline ITM1 & 75 & 46 & $61.33 \%$ & 29 & $38.67 \%$ \\
\hline ATM & 76 & 42 & $55.26 \%$ & 34 & $44.74 \%$ \\
\hline OTM1 & 76 & 33 & $43.42 \%$ & 43 & $56.58 \%$ \\
\hline OTM2 & 76 & 17 & $22.37 \%$ & 59 & $77.63 \%$ \\
\hline Average & & & $49.45 \%$ & & $50.55 \%$ \\
\hline
\end{tabular}

Note. This table presents the conditional probabilities given that bull or bear markets are identified using the MA indicator. The total number of the monthly option trading is 217 , in which about 141 observations (buying calls) are traded in bull markets and 76 observations (buying puts) are traded in bear markets. Each row presents the conditional number and the percentage of options trading having a positive profit or a negative profit given that bull or bear markets are identified. The two percentage columns (\%) are the concept of probabilities.

\section{A New View of Market Trends for Options Trading}

\subsection{The concept}

According to Section 2, an identified market trend using a technical indicator can improve the average performance for the monthly options trading but not for the weekly one. However, the results of Table 6 reveal the underlying problem of classical trend definitions to options trading: A trend can only be determined in hindsight because the future prices are not predictable! For the monthly options trading with trend identification, although the returns have statistically and 
significantly increased, the chance for options traders to lose is still high. For example, the percentage of losing money for buying calls (puts) given that the market is bullish (bearish) is $40.43 \%$ (44.74\%) for the ATM options and increases to $77.14 \%(77.63 \%)$ for the OTM2 options. Hence, the definitions of classical market trends for the options trading are not so useful because the trends are loosely defined.

As stated in Section 2, the Dow theory defines a bull (bear) market as the stock prices experience a series of successively higher (lower) peaks and higher (lower) troughs during a period, in which the prices commonly rise (fall) by $20 \%$ or more. A long-term bull (bear) market may consist of several major up (down) trends and several minor down (up) trends. These classical definitions may be useful in trading stocks but not in trading options. In practice, the short time frame of options trading, the loose definitions of classical trends, the uncertain future stock prices, and the unique property of time value for options are all responsible for the high probabilities of losing money given that a trend is identified. Therefore, we hereby introduce a new view of market trends that will be consistent with the options trading, which is to simplify the market trends into the concept of uptrend, downtrend ${ }^{16}$, and neutral (or sideways) trend ${ }^{17}$. This concept is illustrated in Figure 2.

16 Since the terms of "bull market" and "bear market" are typically reserved for extended periods in which a large portion of security prices are rising and falling (see Farley, 2020), and the bull and bear markets are also known as the primary markets (see Investopedia, 2021), this paper does not use these terms in order to avoid confusion.

17 The word neutral is generally used to describe a financial instrument that does not move in price. Although this is technically accurate, the word has a slightly broader meaning in the context of options trading. Also, when the price of a security goes up and down by small amounts over a period of time, it is said to be moving sideways. (See "Neutral Market Trading Strategies", https://www.optionstrading.org/strategies/neutral-market/). 


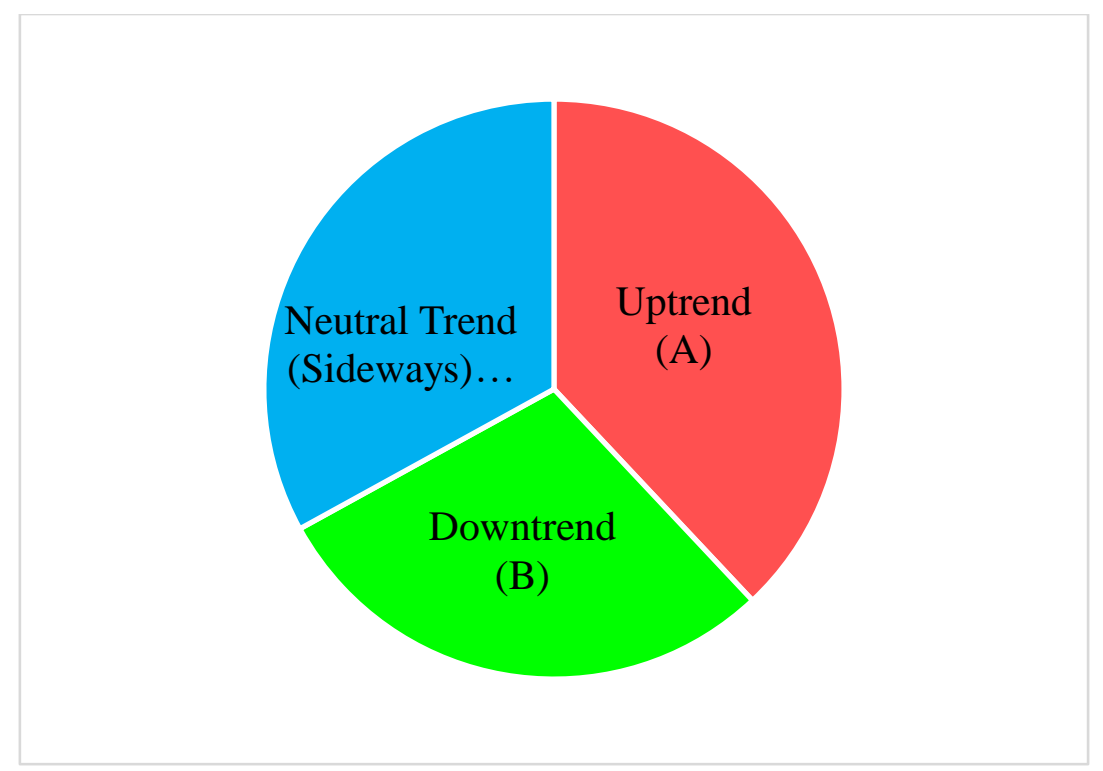

Figure 2: A new concept of market trends

To make positive profits on call options, the market must be truly in an upward trend. More specifically, the necessary condition for a call option with a long position to make a profit is only when the price of underlying asset at time $t=T$ (the expiration date) is more than the strike price plus the call price, i.e., $S_{T}>X+c$. If the ATM option ( $X=S_{0}$, the current stock price) is used, this condition is equivalent to $S_{T}>S_{0}+c$. Thus, the uptrend for options trading in a specific period is defined as when the stock price rises higher than the ATM call premium (i.e., $\Delta S>c$ ) with the same life span. Similarly, the necessary condition for a put option with a long position to make a profit is only when the price of the underlying asset at time $t=T$ is less than the strike price minus the put price, i.e., $S_{T}<X-p$. If the ATM option is used, this condition is equivalent to $S_{T}<S_{0}-p$. Thus, the downtrend for options trading in a specific period is defined as when the stock price falls lower than the ATM put premium (i.e., $\Delta S<-p$ ) with the same life span. When the market is neither an uptrend nor a downtrend, it is a neutral (or sideways) trend, which is defined as $-p<\Delta S<c$. Figure 3 clearly depicts the definitions of the three market trends under the viewpoint of options trading. 


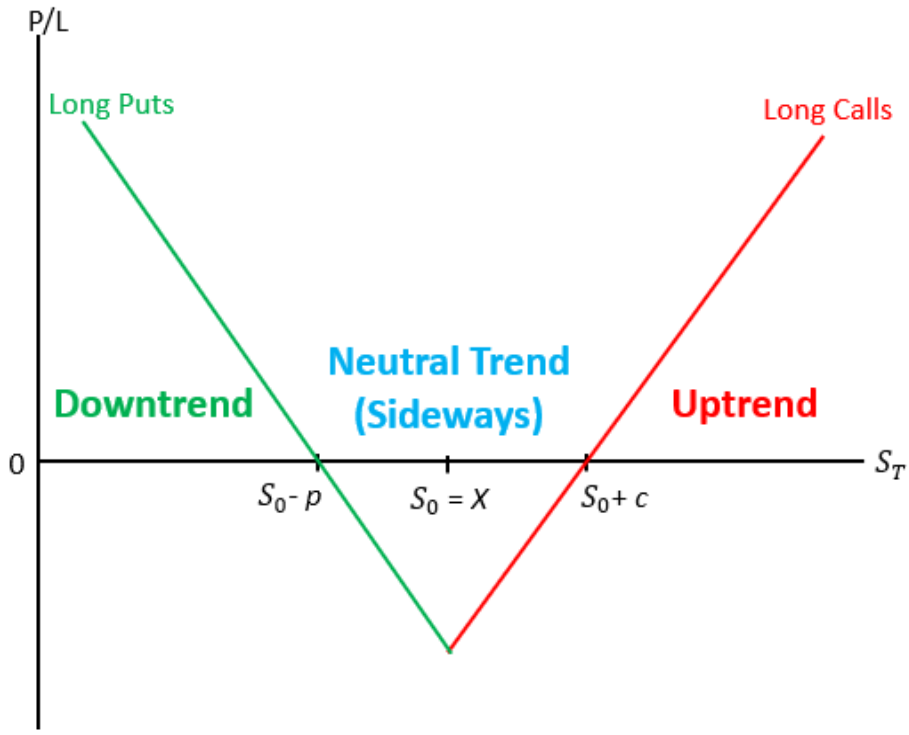

Figure 3: The definitions of market trends for options trading

Note. $S_{0}$ and $S_{T}$ denote the stock prices at time $t=0$ and $T$, the option expiration date; $X, c$, and $p$ are the strike price of option, the call price and the put price, respectively. In the uptrend, the ATM call with long positions can make profits only when the price of underlying asset at time $\mathrm{t}=T$ is more than the strike price plus the call price, i.e., $S_{T}>X+c=S_{0}+c$. In the downtrend, the ATM put with long positions can make profits only when the price of underlying asset at time $t=T$ is less than the strike price minus the put price, i.e., $S_{T}<X-p=S_{0}-p$. When the market is neither an uptrend nor a downtrend, it is a neutral (or sideways) trend, and the stock price is between $S_{0}+c$ and $S_{0}-p$.

In summary, the differences between the classical view and the new view of market trends are as follows: For the classical view of market trends, a bull market is a period of generally rising prices by $20 \%$ or more; a bear market is a general decline of $20 \%$ or more on prices from recent highs over a sustained period; when the price has been experiencing neither an upward trend nor a downward trend, it is a sideways trend, and peaks and troughs continue to be constant. The duration of a market trend (bullish or bearish) may last for several years to decades. In contrast, under the new view of market trends, an uptrend is defined as when the stock price increases over the call critical point, or more specifically, the percentage of the OTM call premium to the initial stock price in the life of a call option (weekly, monthly, or quarterly); a downtrend is defined as when the stock price declines more than the put critical point, or more specifically, the percentage of the OTM put premium to the initial stock price in the life of a put option; a neutral trend is defined as the percentage of the stock price changes between these two critical points in the life of an option. 
Options traders do not know what the trend will be when placing a trading order because the future prices are unknown. Since a trend can only be determined in hindsight, it is interested to know what the probabilities of these three market trends will be for options trading. Therefore, this paper provides the theoretical and empirical estimation methods to discover the probabilities of the market trends.

\subsection{Theoretical estimation of the probabilities of the three market trends}

According to the empirical research of Summa (2003) and the theoretical analysis of Hsu (2015), the options sellers, on average, are the winners. Summa's study is based on analyzing a three-year period of data from the Chicago Mercantile Exchange (CME) for options on futures, while Hsu's analysis can be applied to the stock market. ${ }^{18}$ Therefore, it would be more objective to use Hsu's method to estimate the probabilities of the three trends in the stock market.

\subsubsection{Theoretical estimation with simulated data}

Hsu (2015) demonstrates the probabilities for some representative returns on different maturities of TXO. When the strike price is ATM $(X=8000)$, the probabilities of having positive returns for monthly and weekly call options are $0.3940\left(\operatorname{Prob}\left\{R_{\text {Call }}>0\right\}=1-0.6060\right)$ and $0.3683(=1-0.6317)$; for monthly and weekly put options are $0.2985\left(\operatorname{Prob}\left\{R_{P u t}>0\right\}=1-0.7015\right)$ and $0.3223(=$ $1-0.6777$ ). Since making positive profits for a call (put) option requires the market to be undergoing a real uptrend (downtrend), this means that $S_{T}>X+c=S_{0}+c$ for a long call as well as $S_{T}<X-p=S_{0}-p$ for a long put. Accordingly, it is reasonable to estimate that the probabilities of stock market having an (a) uptrend (downtrend) within the specified periods are 0.3940 (0.2985) for the monthly-term and $0.3683(0.3223)$ for the weekly-term. The probabilities of stock market having a neutral trend under the specified periods are $0.3075(=1-0.3940-0.2985)$ for the monthly-term and $0.3094(=1-0.3683-0.3223)$ for the weekly-term. Table 7 summarizes the theoretical results of the probabilities of stock market trends under the specified periods by using Hsu's method.

Table 7: The probabilities of stock market trends under the specified periods: theoretical estimation with simulated data

\begin{tabular}{|c|c|c|}
\hline Market trend & Monthly-term period & Weekly-term period \\
\hline Uptrend & 0.3940 & 0.3683 \\
\hline Downtrend & 0.2985 & 0.3223 \\
\hline Neutral trend & 0.3075 & 0.3094 \\
\hline
\end{tabular}

18 The major difference between futures options and non-futures options is the expected growth rates $(\mu)$ of their underlying securities. The expected growth rate of non-futures in the real world may take any value; however, the expected growth rate of the futures is zero. (See the proof of Hsu (2015), footnote 12, p. 129.) 


\subsubsection{Theoretical estimation with real data}

To verify the accuracy of the results of Table 7 based on Hsu's (2015) paper, the theoretical estimation with parameters obtained from the real data is used to estimate the probabilities of stock market trends. In the sampling period of December 2001 through December 2019, the average stock price of TAIEX is 7840, the growth rate of the underlying asset is $7 \%$, the volatility of the stock price is 0.19 , and the risk-free rate ${ }^{19}$ is $1.48 \%$. Hence, the parameters are as follows: $S_{0}=7840$, $\mu=7 \%, \sigma=0.19, r=1.48 \%$. When the strike price is ATM $(X=7840)$, the probabilities of having positive returns for monthly and weekly call options are 0.3807 and 0.3620 ; for monthly and weekly put options are 0.3107 and 0.3283 . Therefore, the probabilities of the stock market having an (a) uptrend (downtrend) within the specified periods are 0.3807 (0.3107) for the monthly-term and 0.3620 $(0.3283)$ for the weekly-term. The probabilities of stock market having a neutral trend under the specified periods are 0.3086 for the monthly-term and 0.3097 for the weekly-term. Table 8 summarizes the theoretical results of the probabilities of stock market trends under the specified periods by using the real data from the whole sampling period.

Table 8: The probabilities of stock market trends under the specified periods: theoretical estimation with real data

\begin{tabular}{|c|c|c|}
\hline Market trend & Monthly-term period & Weekly-term period \\
\hline Uptrend & 0.3807 & 0.3620 \\
\hline Downtrend & 0.3107 & 0.3283 \\
\hline Neutral trend & 0.3086 & 0.3097 \\
\hline
\end{tabular}

\subsection{Empirical estimation}

\subsubsection{Methodology}

As it can be clearly seen in Figure 3, the breakeven points of trading the long ATM calls and the long ATM puts are $S_{0}+c$ and $S_{0}-p$, respectively. In other words, the necessary condition for not losing money on trading the ATM options is that the stock price changes (up or down) must be at least greater than the premiums of the ATM options (i.e., $c$ or $p$ ). The long straddle strategy which is to buy an ATM call and an ATM put is employed here and used to calculate the breakeven points for the monthly and weekly options, respectively. By doing so, the upper limit $S_{0}+c$ as well as the lower limit $S_{0}-p$ is formed. Next, the growth rates (or percentages of change) of the underlying asset price are calculated for different specified periods, monthly-term and weekly-term, respectively. When the growth rate is greater than the upper limit, it is an uptrend; less than the lower limit, a downtrend; between the upper and lower bound, a neutral trend.

19 This is the average one-year fixed deposit rate obtained from the five major banks in Taiwan: Bank of Taiwan, Taiwan Cooperative Bank, Land Bank of Taiwan, Hua Nan Bank, and First Commercial Bank. 


\subsubsection{Data and the model}

To analyze the Taiwan stock market trends and calculate the growth rate of the TAIEX, this paper adopts the data of the TAIEX futures (TXF) whose underlying asset is the TAIEX. The reason for using the TXF data here is that TXF pays nonuniform cash dividends, especially in the June-August period, and more importantly, the futures price already reflects the ex-dividend of the stock price. The sampling period of the TXF covers December 2001 through December 2019 for the monthly futures as well as August 2013 through December 2019 for the weekly futures. ${ }^{20}$ The settlement prices of the monthly and weekly futures were retrieved from the Taiwan Futures Exchange. The total number of samples were 217 and 331 for the monthly futures and the weekly futures, respectively. The formula used to calculate the growth rate $(\mu)$ of the TXF for the specified periods is $\mu=\left(F_{T}-F_{0}\right) / F_{0}$, where $F_{0}$ and $F_{T}$ are the futures settlement prices at $t=0$ (the time of contract initiation) and $T$ (the time of contract expiration), respectively.

\subsubsection{Results}

\subsubsection{The breakeven points for the options trading vs. the growth rate of the underlying asset}

The breakeven points (or the critical points) of an ATM straddle strategy for the specific life of the TXO are as follows: For the weekly options, the upper limit, $S_{0}+c$, greater than $S_{0}$ is $0.70 \%\left(=c / S_{0}\right)$; the lower limit, $S_{0}-p$, lower than $S_{0}$ is $-0.71 \%\left(=-p / S_{0}\right)$ during the sampling period of August 2013 through December 2019. It means that options traders can make a positive profit when the growth rates of the underlying asset price exceed the range of $-0.71 \%$ and $0.7 \%$. In other words, if the growth rate is greater (less) than $0.7 \%(-0.71 \%)$, then the market is experiencing an (a) uptrend (downtrend) and options traders with a long position of the ATM calls (puts) will be profitable; if the growth rate is between $-0.71 \%$ and $0.70 \%$, then the market is in a neutral trend and options traders will be able to make a profit by selling an ATM straddle. Similarly, for the monthly options, the upper limit is $2.24 \%$ and the lower limit is $-2.31 \%$ during the sampling period of December 2001 through December 2019.

\subsubsection{The probabilities of the market trends for options trading}

The critical points for defining the three trends have been empirically determined as shown in the above subsection. We then employ the same data to estimate the probabilities of the three trends which may occur for options trading during the life of option. Table 9 shows the empirical results of the distributions of the growth rates of the TAIEX for the specific life of options in the sampling period. In panel A of Table 9 for the weekly options, there are 122 observations above the upper limit (the upper critical point), 78 observations below the lower limit (the lower critical

${ }^{20}$ In Taiwan, the monthly TXF and the weekly TXF are launched on December 20, 2001 and August 7, 2013, respectively. 
point), and 130 observations between the two critical points. The probability of the price change greater (less) than the upper (lower) limit is $36.97 \%$ (23.64\%). It equivalently shows that the probabilities of the uptrend, downtrend, and neutral trend for the weekly-term period are $36.97 \%, 23.64 \%$, and $39.39 \%$, respectively. Similarly, in panel B of Table 9 for the monthly options, it reveals that the probabilities of the uptrend, downtrend, and neutral trend for the monthly-term period are $39.35 \%, 26.39 \%$, and $34.26 \%$, respectively.

Table 9: The distributions of the growth rates of the TAIEX for options with a specific life

\begin{tabular}{|c|c|c|c|c|}
\hline \multicolumn{5}{|c|}{ Panel A: Weekly options } \\
\hline Growth rate & $\boldsymbol{\mu}<-\mathbf{0 . 7 1 \%}$ & $\mathbf{- 0 . 7 1 \%} \leq \boldsymbol{\mu} \leq \mathbf{0 . 7 0} \%$ & $\boldsymbol{\mu}>\mathbf{0 . 7 0 \%}$ & Total \\
\hline No. & 78 & 130 & 122 & 330 \\
\hline Prob. & $23.64 \%$ & $39.39 \%$ & $36.97 \%$ & $100 \%$ \\
\hline \multicolumn{5}{|c|}{ Panel B: Monthly options } \\
\hline Growth rate & $\boldsymbol{\mu}<-\mathbf{2 . 3 1 \%}$ & $\mathbf{- 2 . 3 1 \%} \leq \boldsymbol{\mu} \leq \mathbf{2 . 2 4 \%}$ & $\boldsymbol{\mu}>\mathbf{2 . 2 4 \%}$ & Total \\
\hline No. & 57 & 74 & 85 & 216 \\
\hline Prob. & $26.39 \%$ & $34.26 \%$ & $39.35 \%$ & $100 \%$ \\
\hline
\end{tabular}

Note. This table presents the distributions of the growth rates $(\mu)$ under the given breakeven points of the weekly options and the monthly options, respectively.

The empirical results of the probabilities of the three market trends for options trading are summarized in Table 10.

Table 10: The probabilities of Taiwan stock market trends under the specified periods: an empirical estimation

\begin{tabular}{|c|c|c|}
\hline Market trend & Monthly-term period & Weekly-term period \\
\hline Uptrend & 0.3935 & 0.3697 \\
\hline Downtrend & 0.2639 & 0.2364 \\
\hline Neutral trend & 0.3426 & 0.3939 \\
\hline
\end{tabular}

\subsection{The comparison between the theoretical analysis and the empirical estimation}

The probabilities of the market trends for options trading by using different methods to estimate have been discussed in Section 3.2 and 3.3. We list the comparison of the results between the theoretical analysis and the empirical estimation in Table 11 and note the following features:

a) Both the theoretical and empirical results of the probabilities of an uptrend are consistent with each other. The probability is close to $40 \%$ for the monthly-term period, and around $37 \%$ for the weekly-term period. 
b) All the probabilities of a downtrend are less than that of an uptrend. The probabilities of a downtrend are around 30\% and $25 \%$ for the theoretical and empirical results, respectively.

c) The probabilities of a neutral trend are all above $30 \%$ and almost one third of the whole market trend.

Table 11: The comparison of the probabilities of the market trends under the specified periods

\begin{tabular}{|c|c|c|c|c|c|c|}
\hline & \multicolumn{3}{|c|}{ Monthly-term period } & \multicolumn{3}{c|}{ Weekly-term period } \\
\hline $\begin{array}{c}\text { Market } \\
\text { Trend }\end{array}$ & $\begin{array}{c}\text { Theoretical } \\
\text { prob. (1) }\end{array}$ & $\begin{array}{c}\text { Theoretical } \\
\text { prob. (2) }\end{array}$ & $\begin{array}{c}\text { Empirical } \\
\text { prob. }\end{array}$ & $\begin{array}{c}\text { Theoretical } \\
\text { prob. (1) }\end{array}$ & $\begin{array}{c}\text { Theoretical } \\
\text { prob. (2) }\end{array}$ & $\begin{array}{c}\text { Empirical } \\
\text { prob. }\end{array}$ \\
\hline Uptrend & $39.40 \%$ & $38.07 \%$ & $39.35 \%$ & $36.83 \%$ & $36.20 \%$ & $36.97 \%$ \\
\hline Downtrend & $29.85 \%$ & $31.07 \%$ & $26.39 \%$ & $32.23 \%$ & $32.83 \%$ & $23.64 \%$ \\
\hline $\begin{array}{c}\text { Neutral } \\
\text { trend }\end{array}$ & $30.75 \%$ & $30.86 \%$ & $34.26 \%$ & $30.94 \%$ & $30.97 \%$ & $39.39 \%$ \\
\hline
\end{tabular}

\section{Implications}

\subsection{Revisiting the new view of the market trends and its implications}

In this paper, a new framework of analyzing the market trends for the benefit of trading options has been suggested as stated in Section 3.1. The differences between the classical view and our view of the market trends are as follows:

The first one is the magnitudes of stock price changes for the market trends. Under the classical view, a bull market is a period of price rising by generally $20 \%$ or more; a bear market is a general decline on price from recent highs over a sustained period by $20 \%$ or more; a correction is when the price has been experiencing neither an upward trend nor a downward trend. In contrast, for the new view, an uptrend (downtrend) is defined as when the stock price increases (declines) more than the call (put) critical point, which is a percentage of the OTM call (put) premium to the initial stock price occurring in the life of a call (put) option, which is usually only a small percentage; a neutral trend is defined as when the stock price only changes between the two critical points during the life of an option.

The second one is the duration of trend periods. For the classical view, the duration of a market trend (bullish or bearish) may last for several years to decades. In contrast, for the new view, the duration is only a short time frame (weekly, monthly, or quarterly), depending on options trading.

The third one is the identification of the market trends. Under the classical view, market trends are usually identified by technical indicators; however, under our view, market trends are not easy to identify for options trading because the future 
stock prices are unknown, therefore the occurrence of market trends can only be represented in probabilities. Accordingly, the usefulness of a technical analysis for identifying trends in options trading is doubtful. If a new method of identifying trends in the market fluctuation can be discovered in the future, it will bring great benefits to the practice of trading options as well as academia.

Moreover, the most important implication of the new concept of the market trends is that traders will have consistent results of trading options throughout various market trends: Traders who have a long ATM call during an uptrend, a long ATM put during a downtrend, or a short ATM straddle during a neutral trend will have greater certainty about making a profit and will never be confused again!

\subsection{Options trading strategies applied in the specific markets}

Options trading has a multitude of strategies. According to the new definition of the market trends, each market trend has appropriate options trading strategies and less confusion for the implementation. Here, the commonly used strategies are reclassified as upward trend strategies, downward trend strategies, and neutral trend strategies.

\subsubsection{Upward trend strategies}

In the long run, the worldwide stock market shows a general upward rise, and the probabilities of uptrends under the specified periods are almost close to $40 \%$ which account for most of the proportion as shown in Section 3.4. The options trading strategies commonly applied in an uptrend involve the Long Call, Short Put, Covered Call, Protective Put, Protective Collar, Bull Call Spread, Bull Put Spread, Call Ratio Backspread, and so on. Each bullish options trading strategy comes with its own unique characteristics. For example, purchasing a call can get the maximum potential profits but requires a higher capital; buying a Bull Call Spread can reduce the investment cost, but it has the negative effect of a time value decay as well as the potential profits are limited.

\subsubsection{Downward trend strategies}

In practice, the probabilities of bear market are less than that of bull market. The probabilities of downtrends under the specified periods shown in Section 3.4 are around $25 \%$ to $30 \%$. The options trading strategies commonly used in a downtrend contain the Long Put, Short Call, Reverse of Covered Call, Reverse of Protective Put, Bear Call Spread, Bear Put Spread, Put Ratio Backspread, and so on. Each bearish options trading strategy also comes with its own unique characteristics. For instance, purchasing a put can get the maximum potential profits but requires a higher cost; a Bear Put Spread, which is buying a put while also writing a put with a lower strike, can reduce the investment cost and offset the negative effect of a time value decay, but the potential profits are limited. 


\subsubsection{Neutral trend strategies}

The probabilities of neutral trends under the specified periods shown in Section 3.4 are more than $30 \%$. Under these circumstances, it is not easy to profit from securities with linear returns since the asset prices change within a narrow window. However, using neutral options trading strategies can be profitable in such markets. Examples include the Time Spread, Ratio Spread, Bull Spread, Bear Spread, Butterfly Spread, Condor Spread, Short Straddle, Short Strangle, Iron Butterfly, Iron Condor, and so on. These strategies are useful when the direction of the stock price movement is unpredictable. Note that some of the strategies, such as the Short Straddle and Short Strangle which stand on the side of sellers, combine huge risk under high market uncertainty. ${ }^{21}$ Although many studies, for example, Summa (2003), Bollen and Whaley (2004), Santa-Clara and Saretto (2009), and Hsu (2015) indicate that the options sellers have a trading edge and thus they on average are the winners, it is still necessary to control the risk or to buy insurance to prevent serious loses. Notice that the insurance for Short Straddle (or Short Strangle) is Iron Butterfly (or Iron Condor), which is additionally to purchase an OTM put option with lower strike and an OTM call option with higher strike.

\section{Conclusion}

A market trend is a perceived tendency of a financial market to move in a particular direction over time. The conventional Dow theory classifies the market trends as: a secular trend for long-term frames, a primary trend for medium-term frames, and a secondary trend for short-term frames. However, the definitions of classical market trends are not very helpful in options trading because in practice, options trading often takes place within a short-term frame. Even in a long or medium term of a bull (bear) market, there is a probability of losing all investment for buying call (put) options and a probability of earning big money from buying put (call) options. Options traders are often puzzled by these incongruences. Besides, the conventional market trends are identified by using technical indicators with historical data which often shows time lags. Since future security prices are not easy to predict, market trends are often only shown in hindsight.

Instead of using the classical view of the market trends, this paper stands on the viewpoint of options trading and simplifies the market trends into three concepts: uptrend, downtrend, and neutral trend. A necessary condition is assumed that not losing money for trading ATM options requires that the change in the stock price (up or down) must be at least greater than the premiums of the ATM options (i.e., $c$ or $p$ ). As an example, a long straddle strategy was employed to calculate the

${ }^{21}$ Nick Leeson, employee of Barings Bank, placed a short straddle in the Singapore and Tokyo stock exchanges on January 16, 1995, essentially betting that the Japanese stock market would not move significantly overnight. However, the Kobe earthquake hit early in the morning on January 17, sending Leeson's trading positions into a tailspin. He attempted to recoup the losses by making a series of increasingly risky new trades and eventually caused the collapse of Barings Bank. https://en.wikipedia.org/wiki/Nick_Leeson. 
breakeven points, with the upper limit, $S_{0}+c$, and the lower limit, $S_{0}-p$. When the growth rate of the underlying asset price is greater (less) than the upper (lower) limit, it is an (a) upward (downward) trend; when the growth rate is between the upper and lower bounds, it is a neutral trend. Since options traders cannot easily identify the market trends during a specific period, (i.e., a trend can only be determined in hindsight), the overall trends (i.e., uptrend, downtrend, and neutral trend) can only be represented by probabilities. Through a theoretical analysis and an empirical estimation, it was found that the probability is close to $40 \%$ for an uptrend; around $30 \%$ and $25 \%$ in the theoretical and empirical results for a downtrend, respectively; above $30 \%$ for a neutral trend.

Options traders should recognize that the market trends are not easy to identify but come across probabilities. If they examine the market trends using this new perspective, they will obtain consistent set of probabilities for having positive profits in terms of various options trading strategies. The traders will never be confused again! Of course, if an improved method of trend identification can be utilized in the future, it will bring greater certainty and higher benefits to options trading in practice as well as in academia.

\section{ACKNOWLEDGEMENTS}

Monica Guling Wu gratefully acknowledges the Department of Money and Banking of National Kaohsiung University of Science and Technology for financial aid. 


\section{References}

[1] Bollen, N. and Whaley, R. (2004). Does Net Buying Pressure Affect the Shape of Implied Volatility Functions? Journal of Finance, 59(2), pp.711-753.

[2] Coval, J. D. and Shumway, T. (2001). Expected option returns. Journal of Finance, 56(3), pp. 983-1009.

[3] Edwards, R. D., Magee, J. and Bassetti, W.H.C. (2018). Technical Analysis of Stock Trends, 11th Edition, CRC Press.

[4] Farley, A. (2020). Are We in a Bull Market or a Bear Market? https://www.investopedia.com/ask/answers/are-we-in-bull-market-or-bearmarket/

[5] Hsu, H. (2015). Options Trading, Buy Side or Sell Side? Theoretical Analysis and Interpretation. Journal of Futures and Options, 8(3), pp. 97-148.

[6] Investopedia (2021). Identifying Market Trends. https://www.investopedia.com/articles/technical/03/060303.asp

[7] Lai, K. H. (2020). Can RSI and MACD Technical Indicators Improve the Performance of Option Trading? An Empirical Study on the Taiwanese Options, Unpublished Master Thesis, Master's Program of Feng Chia University, Taiwan.

[8] Santa-Clara, P. and Saretto, A. (2009). Option Strategies: Good Deals and Margin Calls. Journal of Financial Markets, 12(3), pp. 391-417.

[9] Stevens, L. (2002). Essential Technical Analysis: Tools and Techniques to Spot Market Trends, John Wiley and Sons, New York.

[10] Summa, J. F. (2003). Option Sellers vs. Buyers: Who Wins? Futures, 32(4), pp. 52-55.

[11] Tu, J. L. (2020). Can KD and MA Technical Indicators Improve the Performance of Options Trading? Unpublished Master Thesis, Master's Program of Feng Chia University, Taiwan.

[12] Wikipedia (2019). Market Trend. https://en.wikipedia.org/wiki/ Market_trend

[13] Yardeni, E., Abbott, J. and Quintana, M. (2020). Stock Market Briefing: S\&P 500 Bull \& Bear Market Tables. Yardeni Research, Inc. Retrieved from https://www. yardeni.com/pub/sp500corrbeartables.pdf 